\title{
Racial/ethnic and socioeconomic survival disparities for children and adolescents with central nervous system tumours in the United States, 2000-2015
}

\author{
Hannah K. Mitchell ${ }^{\mathrm{a}, \mathrm{b}, *}$, Melanie Morris ${ }^{\mathrm{a}}$, Libby Ellis ${ }^{\mathrm{a}}$, Renata Abrahão ${ }^{\mathrm{c}, \mathrm{d}}$, Audrey Bonaventure ${ }^{\mathrm{a}, \mathrm{e}}$ \\ ${ }^{\mathrm{a}}$ London School of Hygiene and Tropical Medicine, Keppel St, Bloomsbury, London, WC1E 7HT, UK

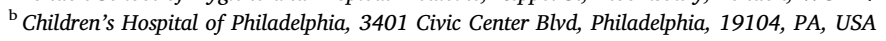 \\ ${ }^{\mathrm{c}}$ Brazilian Cancer Foundation, R. dos Inválidos, 212 - Centro, Rio de Janeiro - RJ, 20231-048, Brazil \\ ${ }^{\mathrm{d}}$ Department of Internal Medicine, Division of Hematology and Oncology, University of California Davis School of Medicine, Sacramento, 4610 X St, Sacramento, 95817, \\ CA, USA \\ ${ }^{\mathrm{e}}$ Epidemiology and Cancer Statistics Group, Department of Health Sciences, University of York, Heslington, York, YO10 5DD, UK
}

\section{A R T I C L E I N F O}

\section{Keywords:}

Childhood cancer

Central nervous system tumour

Race

Ethnicity

Socioeconomic

Survival

Social determinants

SEER

\begin{abstract}
A B S T R A C T
Background and objectives: Central nervous system (CNS) malignancy is the commonest cause of cancer death in children and adolescents (0-19 years) in high-income settings. There is limited data on survival inequalities by race/ethnicity and socioeconomic position (SEP), for young patients, we aim to analyse their influence on survival from childhood CNS tumour.

Methods: 9577 children and adolescents diagnosed with primary malignant CNS tumours during 2000-2015, followed up until Dec 31 st, 2015, and reported to cancer registries (Surveillance, Epidemiology and End Results programme) were included in the analysis. Cox regression models estimated the hazard ratios for race/ethnicity, SEP, and individual insurance status, adjusting for sex, age, diagnostic period, and tumour type. Individual-level insurance status data were available from 2007.

Results: $62.5 \%$ children and adolescents were non-Hispanic White, $10.6 \%$ were non-Hispanic Black and $26.9 \%$ were Hispanic. Race/ethnicity was strongly associated with survival ( $\mathrm{p}<0.001$ ), even after adjusting for SEP, with Black (HR = 1.39 [95 \%CI 1.23-1.58]) and Hispanic children (HR = 1.40 [95 \%CI 1.28-1.54]) having higher hazards of death than White children. This association remained after adjusting for insurance status. There was an apparent positive association between SEP and survival that was largely attenuated after adjustment for insurance status $(\mathrm{p}=0.20)$. Survival was comparable between those privately and Medicaid-insured. Conclusions: Non-Hispanic Black and Hispanic children had lower survival than their White counterparts. This association, not fully explained by differences in SEP, tumour subtype or health insurance, could be related to racially/ethnically-driven barriers to optimal healthcare, warranting further investigation.
\end{abstract}

\section{Introduction}

Cancer is one of the most common causes of childhood mortality in high-income settings. In the United States (US) in 2017, an estimated 15270 new cases of cancer occurred in children (0-14 years) and adolescents (15-19 years), with 1790 deaths in these age groups [1]. Central nervous system (CNS) tumours are the second most common group of cancers diagnosed in children after leukemia, yet they represent the first cause of death from childhood cancer in the US [2].

Whilst the US has seen impressive gains in cancer survival for children and adolescents over the last few decades, the improvement has not been equal across the population $[3,4]$. Disparities in survival between Black and White children diagnosed with leukemia were first reported in the literature in the 1970s [5]. Over the last 50 years, disparities have recurrently been described across racial/ethnic groups for a range of childhood and adolescent cancers, most notably for acute lymphoblastic leukemia (ALL) [3,5-8]. Previous studies yielded conflicting results on the relation between socioeconomic position (SEP) and survival from childhood and adolescent cancer [9]; a more recent literature review of the relation between parental SEP and survival for

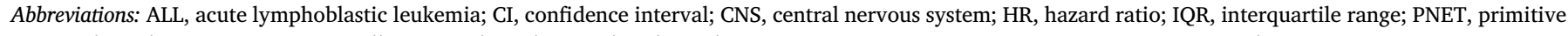
neuroectodermal tumour; SEER, Surveillance, Epidemiology and End Results programme; SEP, socioeconomic position; US, United States

* Corresponding author at: Residency Programme, Children's Hospital of Philadelphia, 3401 Civic Center Blvd, Philadelphia, PA, 19104, USA.

E-mail address: mitchellhk@email.chop.edu (H.K. Mitchell).
} 
childhood cancer in high income settings, including the US and several European countries, reported heterogeneous methods and results, and discussed the potential underlying mechanisms [10]. For childhood CNS tumour more specifically, data on the influence of race/ethnicity on survival are also conflicting, with several US studies reporting lower survival in non-White racial/ethnic groups [8,11-13], but one study reporting no disparities [14]. Using a mediation analysis, Kehm and colleagues found that the racial disparities in CNS tumour survival between 2000 and 2011 were not mediated by SEP [13]. The most recent of these studies, based on data covering years 2001-2008 reported a higher hazard of death for non-Hispanic black patients than non-Hispanic white ones (HR $=1.24$ [95 \% Confidence Interval 1.10-1.40]) but the association was not as clear for Hispanic patients (HR 1.14 [0.94-1.38]) [12].

With regards to SEP, survival disparities by SEP have been reported in adults diagnosed with CNS tumour in a high income setting [15-17], but data are sparse for children. One study reported poorer survival in lower socio-economic groups in Texas [11]. Studies in South Korea and Switzerland showed an adverse effect of low SEP on survival $[18,19]$, however a British study did not show an effect of SEP on survival from CNS tumour in children [20].

Using recent cancer registry data from the SEER (Surveillance, Epidemiology, and End Results) programme covering years 2000-2015, this study examines survival disparities by race/ethnicity and SEP, in a large US population-based cohort of children and adolescents with CNS tumour.

\section{Materials and methods}

\subsection{Study design}

This is a population-based cohort study comprised of routinelycollected cancer registry data from the SEER Programme of the National Cancer Institute (Nov 2017 data release). Since 2000, SEER has included 18 registries ("SEER 18") covering an estimated $34.6 \%$ of the US population. All children and adolescents aged $0-19$ years at diagnosis (hereafter referred to as 'children') with a first primary malignant CNS tumour diagnosed between Jan 1, 2000 and Dec 31, 2015 and residing in the areas covered by SEER 18, were included.

Information about the vital status was collected by SEER registries from routine linkage to the national death index database. Follow-up time for overall survival was computed as the number of days between the date of diagnosis and the earliest of: date of death from any cause, date of last contact (if censored alive), or end date of the study period (Dec 31, 2015). Death was considered irrespective of the cause, as there are few competing causes of death in this age group in high-income countries.

\subsection{Information on race/ethnicity and SEP}

Race was recorded by the SEER registries from self-reported/guardian identification, documentation in the medical records, or death certificates [21]. Hispanic ethnicity was derived by SEER from an algorithm based on the North American Association of Central Cancer Registries (NAACCR) Hispanic Identification Algorithm [22]. We considered three groups of race/ethnicity: non-Hispanic White (referred to as 'White'), non-Hispanic Black (referred to as 'Black'), and Hispanic. Other races/ethnicities were not included due to small numbers.

The SEP measure was based on the area of residence at diagnosis, at the census tract level (typical population size 4000-8000). The Yost Index is constructed by SEER using a factor analysis of seven socioeconomic variables [23,24] including: median household income, median house value, median rent, percent below $150 \%$ of poverty line, education index, percent working class, and percent unemployed. The composite score was grouped into quintiles of the national distribution, with the 1st quintile being people living in the most deprived areas, and

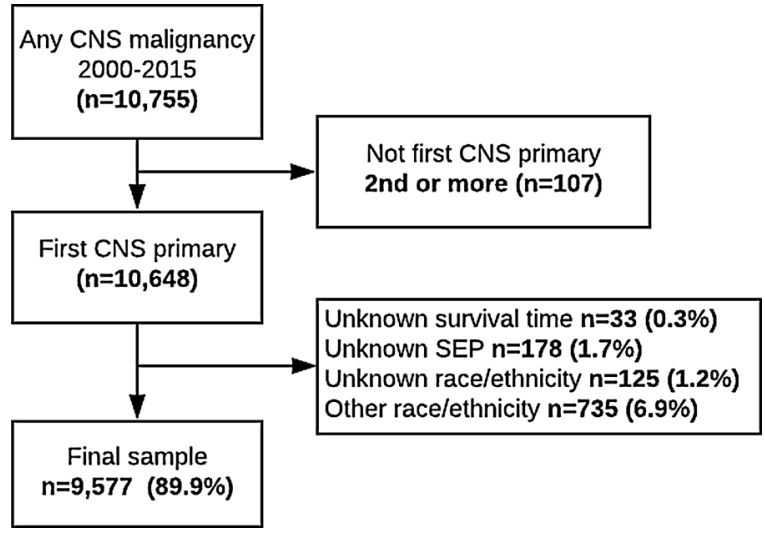

Fig. 1. Flow chart of exclusions from study.

the 5th quintile those in the least deprived areas. From 2007 onwards, individual-level data on health insurance status were also available; classified by SEER as privately insured, insured through Medicaid (state-funded), unspecified insurance, uninsured, or unknown.

Data were retrieved from a case listing session of SEER 18 (November 2017 submission), using SEER*Stat software version 8.3.5. A total of 10755 malignant CNS tumours were recorded in 10,648 children during 2000-2015 (see Fig. 1). When a patient had several records of a CNS tumour, we kept the earliest one (excluding 107 records). We excluded those with missing data on SEP $(n=178)$, on race/ethnicity $(n=125)$, or with a category of 'other race' $(n=735)$, and those whose survival time could not be calculated due to missing date of diagnosis or those reported dead with missing date of death ( $\mathrm{n}=33$ ). A total of 9577 children with a CNS primary tumour were included in the analyses ( $89.9 \%$ of the eligible cohort).

Histological information was recorded and available for all tumours, and six groups of childhood brain tumour were created based on prognostic groupings described previously [14,25]. Information on stage at diagnosis (Summary stage 2000) was available for $93.5 \%$ of the cohort, and classified as localised, regional or distant.

\subsection{Statistical methods}

We tested the proportional hazards assumption graphically with plots of the log-cumulative hazard and Schoenfeld residual plots, and with formal proportional hazard test ( $\mathrm{p}=0.07$ for the race/ethnicity and SEP). Overall survival probabilities at 1 and 5 years after diagnosis were calculated using the Kaplan-Meier method. In order to measure the effect of race/ethnicity and SEP on survival, hazard ratios (HR) and their $95 \%$ confidence intervals (CI) were computed from multivariable Cox proportional hazards models, with all models being adjusted for sex, age, year of diagnosis and tumour category. Additionally, we constructed two sets of models, first analysing race/ethnicity and SEP separately and then together, to assess their potential confounding effect on each other's relation to the hazard of death. We also assessed whether there was an interaction between race/ethnicity and SEP, by adding an interaction term in the final model. Finally, with data on insurance status available from 2007 onwards, a further model was built restricted to patients diagnosed from 2007 (5429 participants). Sensitivity analyses were performed by additionally adjusting the final models for stage at diagnosis (excluding 624 children with missing stage information), and by adding an interaction term between time and race/ethnicity and SEP.

Data were analysed using Stata version 15 (College Station, Texas, USA). 
Table 1a

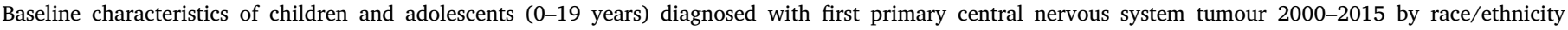
(n = 9577).

\begin{tabular}{|c|c|c|c|c|c|c|c|c|c|}
\hline & & \multicolumn{2}{|c|}{ Non-Hispanic white } & \multicolumn{2}{|c|}{ Non-Hispanic Black } & \multicolumn{2}{|c|}{ Hispanic } & \multicolumn{2}{|r|}{ Total } \\
\hline & & $\mathrm{n}$ & $(\%)$ & $\mathrm{n}$ & $(\%)$ & $\mathrm{n}$ & $(\%)$ & $\mathrm{n}$ & $(\%)$ \\
\hline Total & & 5982 & 62.5 & 1015 & 10.6 & 2580 & 26.9 & 9577 & 100.0 \\
\hline \multirow[t]{5}{*}{ Age at diagnosis (years) } & $<1$ & 287 & 4.8 & 60 & 5.9 & 178 & 6.9 & 525 & 5.5 \\
\hline & $1-4$ & 1574 & 26.3 & 264 & 26 & 777 & 30.1 & 2,615 & 27.3 \\
\hline & $5-9$ & 1574 & 26.3 & 280 & 27.6 & 762 & 29.5 & 2,616 & 27.3 \\
\hline & $10-14$ & 1368 & 22.9 & 235 & 23.2 & 481 & 18.6 & 2,084 & 21.8 \\
\hline & $15-19$ & 1179 & 19.7 & 176 & 17.3 & 382 & 14.8 & 1737 & 18.1 \\
\hline \multirow[t]{2}{*}{ Sex } & Male & 3248 & 54.3 & 500 & 49.3 & 1,381 & 53.5 & 5,129 & 53.6 \\
\hline & Female & 2734 & 45.7 & 515 & 50.7 & 1,199 & 46.5 & 4,448 & 46.4 \\
\hline \multirow[t]{4}{*}{ Year of diagnosis } & $2000-2003$ & 1594 & 26.6 & 252 & 24.8 & 607 & 23.5 & 2,453 & 25.6 \\
\hline & 2004-2007 & 1428 & 23.9 & 209 & 20.6 & 650 & 25.2 & 2,287 & 23.9 \\
\hline & $2008-2011$ & 1535 & 25.7 & 275 & 27.1 & 663 & 25.7 & 2,473 & 25.8 \\
\hline & $2012-2015$ & 1425 & 23.8 & 279 & 27.5 & 660 & 25.6 & 2,364 & 24.7 \\
\hline \multirow[t]{5}{*}{ SEP (Yost) quintile } & 5 least deprived & 1761 & 29.4 & 80 & 7.9 & 245 & 9.5 & 2,086 & 21.8 \\
\hline & 4 & 1437 & 24 & 157 & 15.5 & 382 & 14.8 & 1,976 & 20.6 \\
\hline & 3 & 1181 & 19.7 & 192 & 18.9 & 493 & 19.1 & 1,866 & 19.5 \\
\hline & 2 & 941 & 15.7 & 220 & 21.7 & 636 & 24.7 & 1,797 & 18.8 \\
\hline & 1 most deprived & 662 & 11.1 & 366 & 36.1 & 824 & 31.9 & 1,852 & 19.3 \\
\hline \multirow[t]{6}{*}{ Tumour type } & Astrocytoma (low grade) & 1918 & 32.1 & 286 & 28.2 & 718 & 27.8 & 2922 & 30.5 \\
\hline & Astrocytoma (high grade) & 436 & 7.3 & 94 & 9.3 & 216 & 8.4 & 746 & 7.8 \\
\hline & Astrocytoma (other) & 449 & 7.5 & 82 & 8.1 & 182 & 7.1 & 713 & 7.4 \\
\hline & Ependymoma & 410 & 6.9 & 84 & 8.3 & 264 & 10.2 & 758 & 7.9 \\
\hline & Medulloblastoma/PNET & 1020 & 17.1 & 141 & 13.9 & 510 & 19.8 & 1,671 & 17.4 \\
\hline & Other & 1749 & 29.2 & 328 & 32.3 & 690 & 26.7 & 2,767 & 28.9 \\
\hline \multirow[t]{4}{*}{ Stage } & Localised & 4655 & 77.8 & 771 & 76.0 & 1,911 & 74.1 & 7,337 & 76.6 \\
\hline & Regional & 613 & 10.3 & 113 & 11.1 & 348 & 13.5 & 1,074 & 11.2 \\
\hline & Distant & 325 & 5.4 & 50 & 4.9 & 167 & 6.5 & 542 & 5.7 \\
\hline & Unknown & 389 & 6.5 & 81 & 8.0 & 154 & 5.9 & 624 & 6.5 \\
\hline \multirow[t]{6}{*}{ Type of health insurance (2007 onwards) } & Total & 3336 & 61.5 & 600 & 11.1 & 1,493 & 27.5 & 5429 & 100.0 \\
\hline & Insured (private) & 2111 & 63.3 & 256 & 42.7 & 534 & 35.8 & 2,901 & 53.4 \\
\hline & Insured (Medicaid) & 698 & 20.9 & 251 & 41.8 & 799 & 53.5 & 1,748 & 32.2 \\
\hline & Insured (unknown type) & 389 & 11.7 & 54 & 9 & 101 & 6.8 & 544 & 10 \\
\hline & No insurance & 42 & 1.3 & 19 & 3.2 & 33 & 2.2 & 94 & 1.7 \\
\hline & Unknown & 96 & 2.9 & 20 & 3.3 & 26 & 1.7 & 142 & 2.6 \\
\hline
\end{tabular}

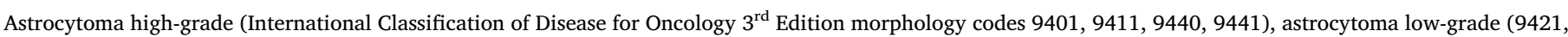

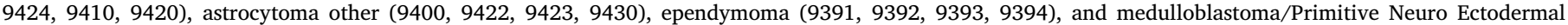
Tumours (PNET) (9470, 9471, 9472, 9364, 9473). All remaining tumours were classified as 'other'.

\section{Results}

Of the 9577 included children diagnosed with a first primary CNS tumour during 2000-2015, $62.5 \%(\mathrm{n}=5982)$ were White, $10.6 \%$ $(\mathrm{n}=1015)$ were Black, and $26.9 \%(\mathrm{n}=2580)$ were Hispanic (Table 1a). Over 3 in 4 participants were aged 1-14 years at diagnosis, with fewer girls than boys ( $46.4 \%$ vs $53.6 \%$ ). Adolescents represented $18.1 \%(n=1737)$ of the study participants. $30.5 \%(n=2922)$ of children had been diagnosed with a low-grade astrocytoma. $7.3 \%$ ( $n=436$ ) of White children had high-grade astrocytoma, the proportion was higher in Black and Hispanic children at $9.3 \%(\mathrm{n}=94)$ and $8.4 \%(\mathrm{n}=216)$, respectively. The vast majority of children $(76.6 \%)$ had localised disease at diagnosis.

The distribution of SEP varied markedly between groups of race/ ethnicity, with more Black and Hispanic children living in the most deprived areas (Chi squared test $\mathrm{p}<0.001$, see Fig. 2). Information on individual-level insurance was available for $97.4 \%$ of the 5429 children diagnosed from 2007 onwards. $20.9 \%(\mathrm{n}=698)$ of White children had Medicaid insurance while the proportion was $41.8 \%(\mathrm{n}=251)$ for Black and $53.5 \%(n=799)$ for Hispanic children $(\mathrm{p}<0.001$, also see Table 1b).

The 9577 children in the cohort contributed to 55052 person-years of follow-up, with a median follow-up time of 56 months (IQR 15-117 months). Overall 1-year survival was $86.8 \%$ (95 \%CI 86.1-87.5 \%) and 5-year survival was $74.5 \%$ (95 \%CI 73.6-75.5 \%) (Table 2). Survival was consistently lower for Black and Hispanic children, than for White children (Fig. 3). One-year survival was 88.8 \% (95 \%CI 87.9-89.6 \%) for White, $84.5 \%(82.1-86.7 \%)$ for Black, and $83.1 \%(81.5-84.5 \%)$ for Hispanic children, and 5-year survival was 78.1 \% (76.9-79.2 \%), $68.6 \%(65.4-71.6 \%)$ and $68.3 \%$ (66.3-70.2\%), for White children, Black and Hispanic children respectively. Children living in the most deprived areas had lower survival than those in the least deprived SEP areas, with 5-year survival of $70.7 \%(68.4-72.9 \%)$ and $78.8 \%$ (76.9-80.7 \%) respectively (Table 2, Fig. 4). Differences in survival were also observed by insurance status (2007-2015) with 5-year survival for privately insured children reaching $76.1 \%(74.2-77.8 \%)$ compared to $70.3 \%(67.7-72.8 \%)$ in children insured on Medicaid (Table 2). Survival was lowest for children diagnosed with high-grade astrocytoma, with 5-year survival of $23.4 \%$ (20.0-26.9 \%). Survival in children with distant disease was 56.6 \% (95 \%CI 51.8-60.7\%) compared to $78.0 \%$ (95 \%CI 77.0-79.0 \%) in those with localised disease.

The crude hazard ratio of death in Black compared to White children was 1.54 (95\%CI 1.36-1.74) (Table 2). When adjusted for age, sex, diagnostic period and tumour type, it was 1.46 (1.29-1.65), and when additionally adjusted for SEP, the hazard ratio for Black children remained stable (1.39 [1.23-1.58]) (Table 3). In the univariable analysis, Hispanic children had increased hazard of death compared to White children (1.50 [1.37-1.65]) (Table 2). When adjusted for age, sex, diagnostic period, and tumour type, the hazard ratio was stable, and it decreased very slightly to 1.40 (95\%CI 1.28-1.54) on additional adjustment for SEP (Table 3). The hazard of death was greatest in the most deprived SEP regions, with evidence of a linear trend with increasing degree of deprivation $(\mathrm{p}<0.002$ for test for trend, $\mathrm{p}=0.6$ for test of departures from linearity). After adjustment for age, sex, 

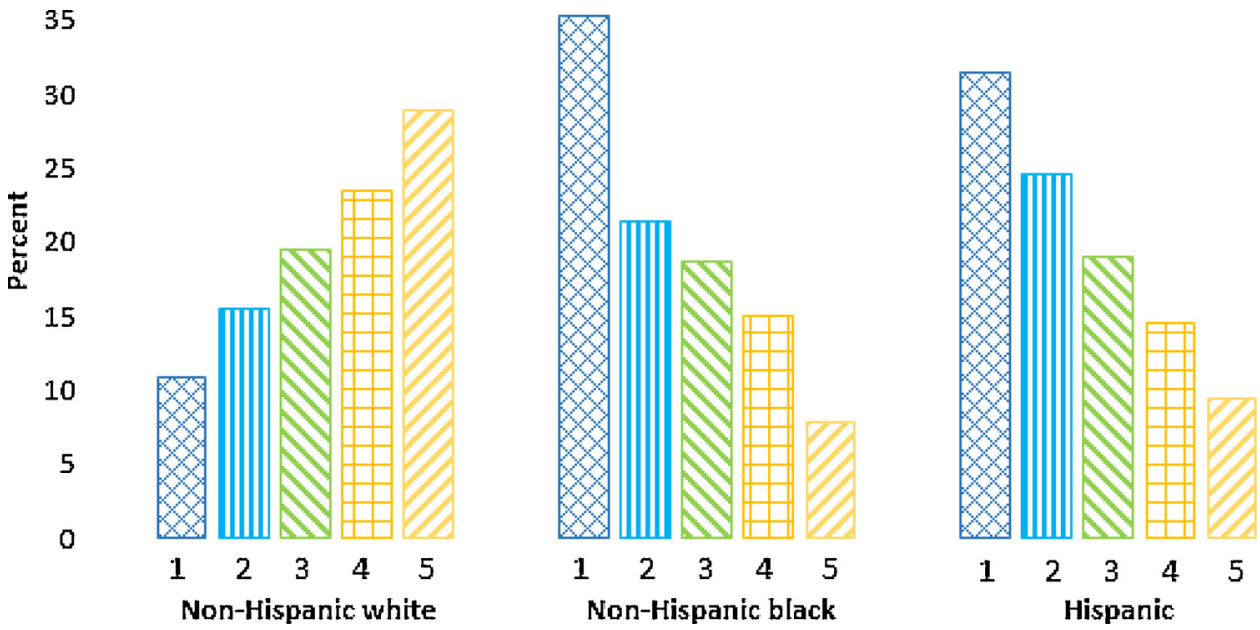

Fig. 2. Distribution of SEP quintiles $(1=$ most deprived, $5=$ least deprived $)$ by race/ethnicity among children and adolescents diagnosed with CNS tumour during 2000-2015 in the US $(\mathrm{p}<0.001)$.

diagnostic period, and tumour type, the hazard of death was 1.46 (1.29-1.66) times higher in children living in the most deprived versus those in the least deprived SEP regions (Table 3). When additionally adjusted for race/ethnicity however, the hazard ratio decreased to 1.23 (95\%CI 1.08-1.41).

When restricting to the 5429 children diagnosed from 2007 onwards, the hazard of death in the Medicaid-insured group was 1.18 (95 \%CI 1.04-1.34) times higher compared to those privately insured (adjusted for age, sex, diagnostic period and tumour type) (Table 4). When additionally adjusted for area-level SEP and race/ethnicity, insurance status was no longer significantly associated with the risk of death $(\mathrm{HR}=1.01$ (95 \%CI 0.87-1.16)). Overall, SEP was not associated with the hazard of death when the analyses were additionally adjusted for insurance status $(\mathrm{p}=0.20)$, despite raised HRs in the most deprived groups. The association with race/ethnicity remained strongly significant when accounting for insurance status.

Sensitivity analyses additionally adjusted for stage at diagnosis (excluding 624 participants) showed similar results (not shown).

Table 1b

Baseline characteristics of children and adolescents (0-19) diagnosed with first primary CNS tumour 2000-2015 by SEP ( $\mathrm{n}=9577)$.

\begin{tabular}{|c|c|c|c|c|c|c|c|c|c|c|c|c|c|}
\hline & & \multicolumn{2}{|c|}{1 (most deprived) } & \multicolumn{2}{|r|}{2} & \multicolumn{2}{|r|}{3} & \multicolumn{2}{|r|}{4} & \multicolumn{2}{|c|}{5 (least deprived) } & \multicolumn{2}{|r|}{ Total } \\
\hline & & $\mathrm{n}$ & (\%) & $\mathrm{n}$ & $(\%)$ & $\mathrm{n}$ & (\%) & $\mathrm{n}$ & $(\%)$ & $\mathrm{n}$ & $(\%)$ & $\mathrm{n}$ & (\%) \\
\hline \multirow{3}{*}{ Age at diagnosis (years) } & $1-4$ & 543 & 29.3 & 506 & 28.2 & 513 & 27.5 & 532 & 26.9 & 521 & 25 & 2615 & 27.3 \\
\hline & $5-9$ & 535 & 28.9 & 502 & 27.9 & 476 & 25.5 & 546 & 27.6 & 557 & 26.7 & 2616 & 27.3 \\
\hline & $10-14$ & 362 & 19.5 & 366 & 20.4 & 420 & 22.5 & 438 & 22.2 & 498 & 23.9 & 2084 & 21.8 \\
\hline Sex & Female & 890 & 48.1 & 871 & 48.5 & 841 & 45.1 & 910 & 46.1 & 936 & 44.9 & 4448 & 46.4 \\
\hline \multirow[t]{4}{*}{ Year of diagnosis } & $2000-2003$ & 451 & 24.4 & 488 & 27.2 & 459 & 24.6 & 503 & 25.5 & 552 & 26.5 & 2453 & 25.6 \\
\hline & 2004-2007 & 452 & 24.4 & 403 & 22.4 & 455 & 24.4 & 464 & 23.5 & 513 & 24.6 & 2287 & 23.9 \\
\hline & 2008-2011 & 477 & 25.8 & 450 & 25.0 & 478 & 25.6 & 538 & 27.2 & 530 & 25.4 & 2473 & 25.8 \\
\hline & 2012-2015 & 472 & 25.5 & 456 & 25.4 & 474 & 25.4 & 471 & 23.8 & 491 & 23.5 & 2364 & 24.7 \\
\hline \multirow[t]{3}{*}{ Race/Ethnicity } & NH White & 662 & 35.7 & 941 & 52.4 & 1181 & 63.3 & 1437 & 72.7 & 1761 & 84.4 & 5982 & 62.5 \\
\hline & NH Black & 366 & 19.8 & 220 & 12.2 & 192 & 10.3 & 157 & 7.9 & 80 & 3.8 & 1015 & 10.6 \\
\hline & Hispanic & 824 & 44.5 & 636 & 35.4 & 493 & 26.4 & 382 & 19.3 & 245 & 11.7 & 2580 & 26.9 \\
\hline Tumour type & Other & 539 & 29.1 & 490 & 27.3 & 553 & 29.6 & 596 & 30.2 & 589 & 28.2 & 2767 & 28.9 \\
\hline \multirow[t]{4}{*}{ Stage } & Localised & 1390 & 75.0 & 1383 & 76.9 & 1429 & 76.6 & 1494 & 75.6 & 1,641 & 78.7 & 7337 & 76.6 \\
\hline & Regional & 237 & 12.8 & 203 & 11.3 & 206 & 11.0 & 222 & 11.2 & 206 & 9.9 & 1074 & 11.2 \\
\hline & Distant & 112 & 6.1 & 91 & 5.1 & 115 & 6.2 & 127 & 6.4 & 97 & 4.7 & 542 & 5.7 \\
\hline & Unknown & 113 & 6.1 & 120 & 6.7 & 116 & 6.2 & 133 & 6.8 & 142 & 6.8 & 624 & 6.5 \\
\hline \multirow[t]{6}{*}{ Type of health insurance (2007 onwards) } & Total & 1052 & 19.4 & 1022 & 18.8 & 1077 & 19.8 & 1124 & 20.7 & 1154 & 21 & 5429 & 100 \\
\hline & Insured (private) & 295 & 15.9 & 437 & 24.3 & 578 & 31.0 & 723 & 36.6 & 868 & 41.6 & 2901 & 30.3 \\
\hline & Insured (Medicaid) & 621 & 33.5 & 448 & 24.9 & 355 & 19.0 & 231 & 11.7 & 93 & 4.5 & 1748 & 18.3 \\
\hline & Insured (unknown type) & 83 & 4.5 & 84 & 4.7 & 95 & 5.1 & 135 & 6.8 & 147 & 7 & 544 & 5.7 \\
\hline & No insurance & 28 & 1.5 & 18 & 1.0 & 22 & 1.2 & 15 & 0.8 & 11 & 0.5 & 94 & 1 \\
\hline & Unknown & 25 & 1.3 & 35 & 1.9 & 27 & 1.4 & 20 & 1 & 35 & 1.7 & 142 & 1.5 \\
\hline
\end{tabular}

Astrocytoma high-grade (International Classification of Disease for Oncology 3rd Edition morphology codes 9401, 9411, 9440, 9441), astrocytoma low-grade (9421, 9424, 9410, 9420), astrocytoma other (9400, 9422, 9423, 9430), ependymoma (9391, 9392, 9393, 9394), and medulloblastoma/Primitive Neuro Ectodermal Tumours (PNET) (9470, 9471, 9472, 9364, 9473). All remaining tumours were classified as 'other'. 
Table 2

1- and 5-year overall survival and univariable (crude) hazard ratios for death among children and adolescents (0-19 years) diagnosed with a first primary CNS tumour between 2000-2015 ( $\mathrm{n}=9577)$.

\begin{tabular}{|c|c|c|c|c|c|}
\hline & & \multicolumn{2}{|r|}{ Survival probabilities } & \multicolumn{2}{|c|}{ Univariate hazard ratio for death } \\
\hline & & 1-year survival (95 \% CI) & 5-year survival (95 \% CI) & $\mathrm{HR}(95 \% \mathrm{CI})$ & p-value ${ }^{a}$ \\
\hline All & & $86.8(86.1-87.5)$ & $74.5(73.6-75.5)$ & & \\
\hline \multirow[t]{3}{*}{ Race/ethnicity } & NH White & $88.8(87.9-89.6)$ & $78.1(76.9-79.2)$ & 1 (ref) & $<0.0001$ \\
\hline & NH Black & $84.5(82.1-86.7)$ & $68.6(65.4-71.6)$ & $1.54(1.36-1.74)$ & \\
\hline & Hispanic & $83.1(81.5-84.5)$ & $68.3(66.3-70.2)$ & $1.50(1.37-1.65)$ & \\
\hline \multirow[t]{5}{*}{ SEP (Yost) quintile } & 5 least deprived & $84.1(82.3-85.8)$ & $70.7(68.4-72.9)$ & 1 (ref) & $<0.0001$ \\
\hline & 4 & $87.5(86.0-89.0)$ & $75.8(73.7-77.8)$ & $1.17(1.02-1.33)$ & \\
\hline & 3 & $86.6(85.0-88.1)$ & $74.0(71.8-76.1)$ & $1.29(1.13-1.47)$ & \\
\hline & 2 & $85.0(83.2-86.6)$ & $72.5(70.2-74.7)$ & $1.38(1.21-1.57)$ & \\
\hline & 1 most deprived & $84.1(82.3-85.8)$ & $70.7(68.4-72.9)$ & $1.49(1.31-1.70)$ & \\
\hline \multirow[t]{5}{*}{ Age at diagnosis (years) } & $<1$ & $70.6(66.4-74.4)$ & $59.7(55.1-64.0)$ & $1.87(1.60-2.19)$ & $<0.0001$ \\
\hline & $1-4$ & $85.8(84.4-87.1)$ & $74.4(72.6-76.1)$ & $1(\mathrm{ref})^{\mathrm{b}}$ & \\
\hline & $5-9$ & $83.9(82.3-85.3)$ & $71.5(69.6-73.3)$ & $1.14(1.03-1.27)$ & \\
\hline & $10-14$ & $90.9(89.6-92.1)$ & $79.4(77.5-81.2)$ & $0.78(0.69-0.88)$ & \\
\hline & $15-19$ & 92.7 (91.3-93.8) & 77.7 (71.6-76.4) & $0.82(0.73-0.94)$ & \\
\hline \multirow[t]{2}{*}{ Sex } & Male & $86.8(85.8-87.7)$ & $73.7(72.4-75.0)$ & 1 (ref) & 0.09 \\
\hline & Female & $86.9(85.8-87.9)$ & $75.5(74.1-76.8)$ & $0.93(0.86-1.01)$ & \\
\hline \multirow[t]{4}{*}{ Year of diagnosis } & $2000-2003$ & $85.7(84.2-87.0)$ & $73.6(71.8-75.3)$ & 1 (ref) & 0.4 \\
\hline & 2004-2007 & $86.8(85.4-88.2)$ & 74.7 (72.9-76.5) & $0.95(0.85-1.05)$ & \\
\hline & 2008-2011 & $86.8(85.3-88.0)$ & $75.5(73.7-77.2)$ & $0.92(0.82-1.02)$ & \\
\hline & 2012-2015 & $88.2(86.7-89.6)$ & - & $0.95(0.84-1.08)$ & \\
\hline \multirow[t]{6}{*}{ Tumour type $^{c}$} & Astrocytoma (low grade) & $98.2(97.6-98.6)$ & 95.5 (94.7-96.3) & $1(\mathrm{ref})^{\mathrm{b}}$ & $<0.0001$ \\
\hline & Astrocytoma (high grade) & $61.2(57.5-64.8)$ & $23.4(20.0-26.9)$ & $26.16(21.68-31.57)$ & \\
\hline & Astrocytoma (other) & $93.1(91.0-94.8)$ & $83.9(80.8-86.6)$ & $3.51(2.74-4.49)$ & \\
\hline & Ependymoma & $94.9(93.1-96.3)$ & 76.7 (73.1-79.9) & $5.46(4.38-6.81)$ & \\
\hline & Medulloblastoma/PNET & $86.3(84.6-87.9)$ & $68.9(66.4-71.2)$ & $7.84(6.50-9.44)$ & \\
\hline & Other & $78.1(76.4-79.6)$ & $65.9(63.9-67.7)$ & $8.54(7.15-10.21)$ & \\
\hline \multirow[t]{5}{*}{ Type of health insurance (restricted to 2007 onwards) } & Insured (private) & $88.5(87.3-89.7)$ & $76.1(74.2-77.8)$ & $1(\text { ref })^{b}$ & $<0.0001$ \\
\hline & Insured (Medicaid) & $85.6(83.7-87.2)$ & $70.3(67.7-72.8)$ & $1.32(1.17-1.50)$ & \\
\hline & Insured (Unknown type) & 88.5 (85.5-91.0) & $80.9(77.0-84.2)$ & $0.82(0.66-1.02)$ & \\
\hline & No insurance & 86.1 (76.8-91.9) & $76.0(64.7-84.1)$ & $1.03(0.65-1.63)$ & \\
\hline & Unknown & $82.6(74.7-88.2)$ & $75.1(65.9-82.1)$ & $1.28(0.89-1.83)$ & \\
\hline \multirow[t]{3}{*}{ Stage $^{\mathrm{d}}$} & Localised & $89.2(88.4-89.9)$ & $78.0(77.0-79.0)$ & 1 (ref) & $<0.0001$ \\
\hline & Regional & $78.2(75.6-80.6)$ & $60.8(57.6-63.9)$ & $1.97(1.76-2.19)$ & \\
\hline & Distant & $74.9(71.0-78.4)$ & $56.4(51.8-60.7)$ & $2.48(2.16-2.84)$ & \\
\hline
\end{tabular}

a Likelihood ratio (LR) test p-value.

b Reference as largest group.

c Astrocytoma high-grade (International Classification of Disease for Oncology 3rd Edition morphology codes 9401, 9411, 9440, 9441), astrocytoma low-grade

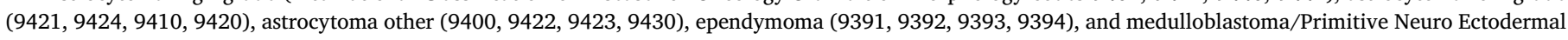
Tumours (PNET) (9470, 9471, 9472, 9364, 9473). All remaining tumours were classified as 'other'.

d Excluding 624 children with unknown stage at diagnosis.

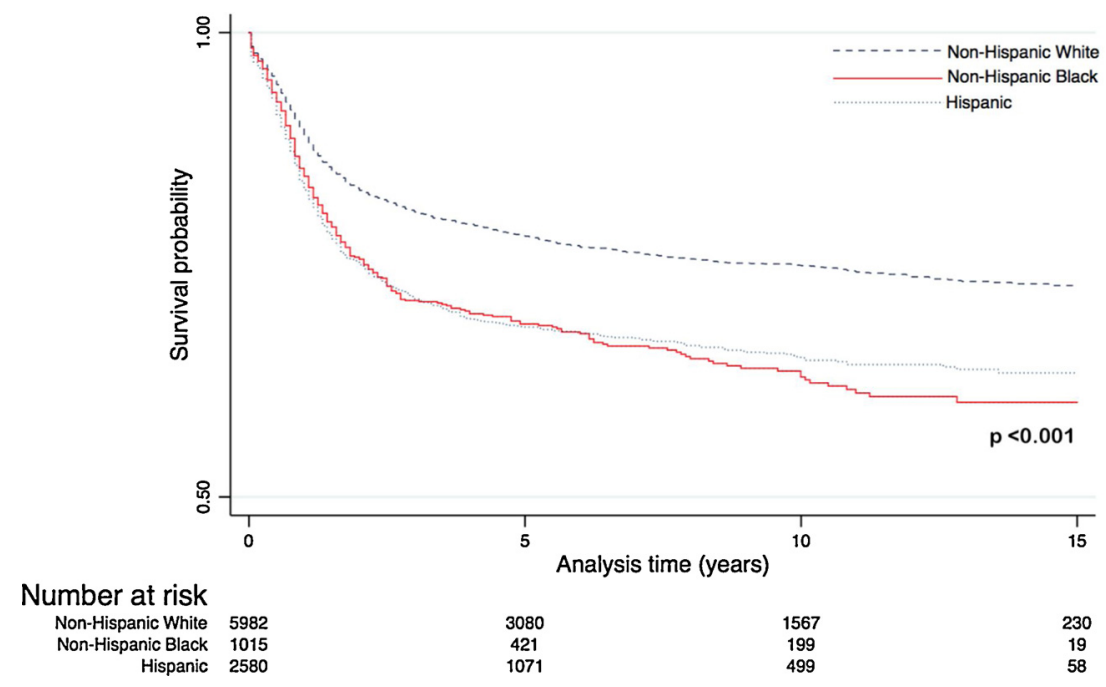

Fig. 3. Kaplan-Meier graph of overall survival of children and adolescents (0-19) diagnosed with a first primary CNS tumour between 2000 and 2015 , by race/ ethnicity.

(Note: $\mathrm{y}$-axis restricted from 0.5 to 1 ). 


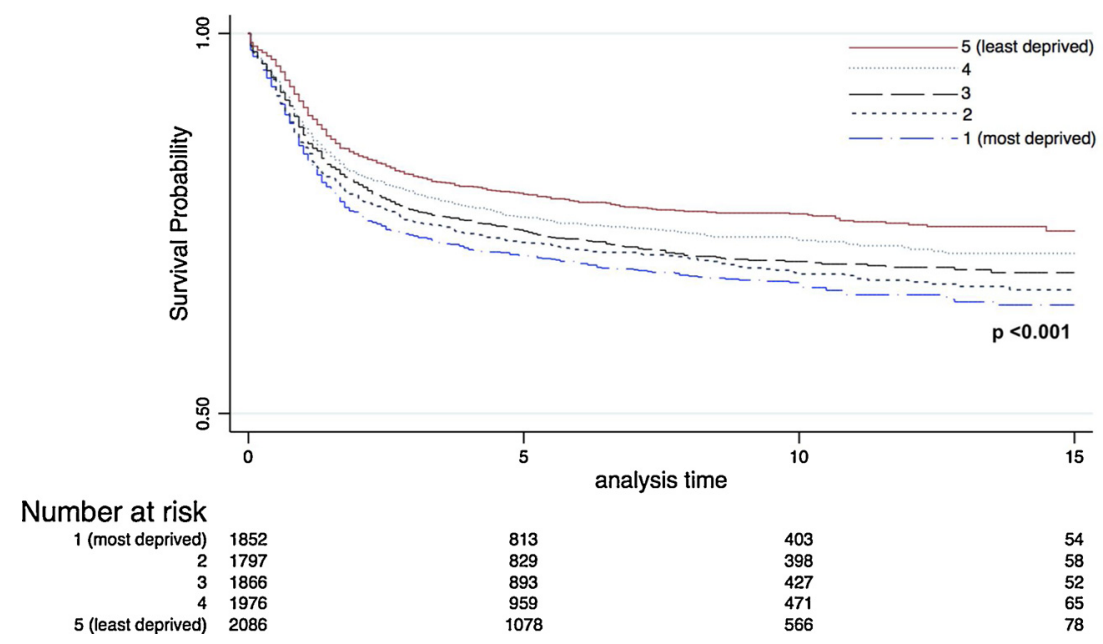

Fig. 4. Kaplan-Meier graph of overall survival of children and adolescents (0-19) diagnosed with a first primary CNS tumour between 2000 and 2015 , by SEP. (Note: y-axis restricted from 0.5 to 1 )

Analyses considering an interaction between race/ethnicity and time since diagnosis (up to or after 2.5 years) showed a slightly smaller association between race/ethnicity and survival for Hispanics after 2.5 years after diagnosis (not shown).

\section{Discussion}

This study used the most recent population-based data available to assess the influence of race/ethnicity and SEP on survival in children and adolescents diagnosed with a CNS tumour in the US. There was a clear relationship between race/ethnicity and SEP, with a higher proportion of Black and Hispanic children residing in the most deprived versus least deprived areas. A substantially higher proportion of White children had private insurance compared to Hispanic and Black children.

We found striking evidence of survival inequalities by racial/ethnic group. Black and Hispanic children had increased hazard of death compared to White children, and this association did not appear to be fully explained by differences in SEP, stage at diagnosis or tumour type, as the effect of race/ethnicity was only slightly attenuated when adjusted for these variables. Conversely, the apparent effect of SEP on survival was markedly attenuated after adjustment for race/ethnicity and insurance status. This suggests that the apparent influence of SEP on survival was largely explained by race/ethnicity and individual-level insurance status. Insurance status was not itself associated with survival.
These findings are based on a large population-based cohort, with high-quality data of the SEER Programme on children diagnosed with CNS tumour over a 15-year period. There is high ascertainment of all incident tumours in the areas covered by SEER, with linkage to death database and low loss to follow-up (approximately $7 \%$ at five years of diagnosis). Detailed demographic and clinical information was available [26]. The measure used to quantify SEP was the Yost Index, which is based on census tract-level socioeconomic indices. As an ecologicallevel variable, this has some limitations, mainly the assumption of homogeneity of people living in the same census tract. Although the census tract-level geographic areas are small, this potential non-differential misclassification means the true effects of SEP on survival might be under-estimated here. However, although some aspects of an individual's SEP might not be completely captured by census level SEP, some research has suggested that both individual and geographic-level measures of SEP have similar association with outcome [27]. Individual-level insurance status data were available for 2007 onwards and this could also serve as a proxy for individual SEP, but this measure does not allow for the capture of differing degrees of socioeconomic disparity.

We analysed the three largest categories of race/ethnicity in the US, but children of mixed race/ethnicity and other minority groups were not included due to small numbers. Potential misclassification of race/ ethnicity obtained by SEER may have influenced the association between race/ethnicity and survival. However, previous studies using US cancer registry data, showed that this variable is of high-quality for self-

Table 3

Adjusted hazard ratios by race/ethnicity and SEP for overall survival of children and adolescents diagnosed with a first primary CNS tumour in SEER data $2000-2015$ $(\mathrm{n}=9577$ in all models).

\begin{tabular}{|c|c|c|c|c|c|}
\hline & & \multicolumn{2}{|l|}{ Partially adjusted $^{\mathrm{a}}$} & \multicolumn{2}{|l|}{ Fully adjusted ${ }^{\mathrm{b}}$} \\
\hline & & aHR $(95 \% \mathrm{CI})$ & p-value ${ }^{c}$ & aHR (95 \% CI) & p-value ${ }^{c}$ \\
\hline \multirow[t]{3}{*}{ Race/ethnicity } & Non-Hispanic White & 1 (ref) & \multirow[t]{3}{*}{$<0.0001$} & 1 (ref) & \multirow[t]{3}{*}{$<0.0001$} \\
\hline & Non Hispanic Black & $1.46(1.29-1.65)$ & & $1.39(1.23-1.58)$ & \\
\hline & Hispanic & $1.48(1.35-1.61)$ & & $1.40(1.28-1.54)$ & \\
\hline \multirow[t]{5}{*}{ SEP (Yost) quintile } & 5 - least deprived & 1 (ref) & \multirow[t]{5}{*}{$<0.0001$} & 1 (ref) & \multirow[t]{5}{*}{$<0.0001$} \\
\hline & 4 & $1.17(1.02-1.33)$ & & $1.13(0.99-1.29)$ & \\
\hline & 3 & $1.29(1.12-1.47)$ & & $1.20(1.05-1.37)$ & \\
\hline & 2 & $1.35(1.18-1.54)$ & & 1.21 (1.06-1.39) & \\
\hline & 1 - most deprived & $1.46(1.29-1.66)$ & & $1.23(1.08-1.41)$ & \\
\hline
\end{tabular}

Final sample includes 624 patients with information missing on stage as this variable was not used in final model.

a Adjusted for sex, age, year of diagnosis and tumour category only.

b Adjusted for sex, age, year of diagnosis and tumour category and other variables in the table (race/ethnicity and SEP).

c Likelihood ratio (LR) test p-value. 
Table 4

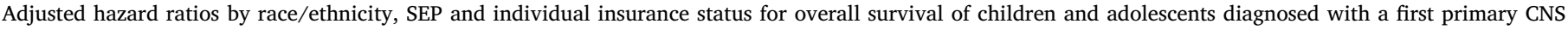
tumour in SEER data, 2007-2015 ( $n=5429$ in both models).

\begin{tabular}{|c|c|c|c|c|c|}
\hline & & \multicolumn{2}{|l|}{ Partially adjusted $^{\mathrm{a}}$} & \multicolumn{2}{|l|}{ Fully adjusted $^{\mathrm{b}}$} \\
\hline & & aHR (95\% CI) & p-value & aHR & p-value ${ }^{c}$ \\
\hline \multirow[t]{3}{*}{ Race/ethnicity } & Non-Hispanic White & 1 (ref) & \multirow[t]{3}{*}{$<0.0001$} & 1 (ref) & \multirow[t]{3}{*}{0.0001} \\
\hline & Non-Hispanic Black & $1.34(1.11-1.60)$ & & $1.25(1.04-1.51)$ & \\
\hline & Hispanic & $1.42(1.25-1.61)$ & & $1.34(1.17-1.54)$ & \\
\hline \multirow[t]{5}{*}{ SEP (Yost) quintile } & 5 least deprived & 1 (ref) & \multirow[t]{5}{*}{0.0007} & 1 (ref) & \multirow[t]{5}{*}{0.2} \\
\hline & 4 & $1.10(0.91-1.33)$ & & $1.07(0.88-1.30)$ & \\
\hline & 3 & $1.25(1.03-1.51)$ & & $1.17(0.96-1.42)$ & \\
\hline & 2 & $1.31(1.08-1.58)$ & & $1.20(0.98-1.46)$ & \\
\hline & 1 most deprived & $1.45(1.20-1.74)$ & & $1.26(1.03-1.55)$ & \\
\hline \multirow[t]{5}{*}{ Type of health insurance (2007 onwards) } & Insured (Private) & 1 (ref) & \multirow[t]{5}{*}{0.007} & 1 (ref) & \multirow[t]{5}{*}{0.2} \\
\hline & Insured (Medicaid) & $1.18(1.04-1.34)$ & & $1.01(0.87-1.16)$ & \\
\hline & Insured (unknown type) & $0.83(0.67-1.03)$ & & $0.82(0.66-1.02)$ & \\
\hline & No insurance & $1.07(0.68-1.69)$ & & $0.97(0.61-1.53)$ & \\
\hline & Unknown & $1.40(0.97-2.01)$ & & $1.36(0.94-1.96)$ & \\
\hline
\end{tabular}

Final sample includes 624 patients with information missing on stage as this variable was not used in final model.

There was no evidence of interaction between race/ethnicity and SEP ( $\mathrm{p}_{\text {int }}=0.2$ ).

a Adjusted for sex, age, year of diagnosis and tumour category only.

b Adjusted for sex, age, year of diagnosis and tumour category and other variables in the table (race/ethnicity, SEP and insurance).

c Likelihood ratio (LR) test p-value.

reported race/ethnicity for White and Black patients and of moderate quality for Hispanic patients [28].

Information on the type of treatment received was not available for the majority of children, and was therefore not analysed. Information on stage at diagnosis was missing for a substantial part of the eligible cohort, and therefore restricted to sensitivity analyses. Future research using these data would be important in elucidating the mechanisms behind survival disparities, as one can hypothesise that being diagnosed at a later stage, or receiving suboptimal treatment, could explain some of the survival inequalities.

Our results confirm the persistence of racial/ethnic disparities in survival of childhood CNS tumour observed up to year 2011 [8,11-13], using a more recent and larger data set. In the US, socioeconomic disparity is often associated with race/ethnicity, warranting the need to consider the two factors together [29]. In 2016, $22 \%$ of the Black population and $19 \%$ of the Hispanic population lived below the poverty line, while the figure was $9 \%$ for the White population [30]. Our study suggests that the association between race/ethnicity and CNS tumour survival in children is, if at all, little confounded by SEP and insurance status. A recent analysis of SEER data covering years 2000-2011 also reported racial/ethnic disparities in survival for several types of childhood cancer, including astrocytomas and non-astrocytoma CNS tumours [13]. In this study, there was no evidence that the association between race/ethnicity and survival for non-astrocytoma CNS tumours was mediated by SEP. The authors discussed the possibility that the effect of race/ethnicity on survival from CNS tumours could be mediated by other factors not encapsulated within area-based SEP, such as differences in tumour biology and other social factors [13]. Our results support the low confounding effect of SEP on the association between race/ethnicity and CNS tumour survival. In addition, this particularly robust association remained stable despite accounting for the differences in distribution of prognostic-based tumour type and stage by race/ethnicity. Whilst, we cannot rule out that other biological and/or genetics differences explain this association, there is evidence from clinical trials at a large US children's cancer hospital showing that, with access to equal treatment for the same type of tumour, Black children with cancer had equivalent survival to White children [31]. Whilst evidence coming from clinical trial data must be interpreted with caution due to racial differences in enrollment [32], these results showing that equal treatment may reduce or eliminate the racial survival disparities among children with cancer, together with our findings, support the hypothesis that the observed racial/ethnic survival disparities could be due to differential access to optimal healthcare.

Individual insurance data has only been collected by SEER since 2007, therefore research investigating its impact is still limited. A US study from 2007 to 2009 found an increased risk of cancer death in uninsured children, however numbers were small [33]. Importantly in our study, comparable survival was seen between children who were privately insured and those who were insured under a Medicaid programme, suggesting that access to insurance, either public or private, might reduce survival inequalities. Results on absence of insurance must still be interpreted with caution, as numbers are small for uninsured children. In addition, misclassification of insurance data has been reported in children who initially presented for healthcare without insurance (therefore, registered as uninsured), but who later obtained insurance, as in many US states, uninsured patients may get Medicaid coverage after a cancer diagnosis [33]. Therefore, the true effect of being uninsured or underinsured on the risk of death could have been underestimated in our study.

It is possible that some personal reasons could lead those without insurance or of lower SEP to present to healthcare at a more advanced stage of disease, such as fear of medical costs or fear of legal repercussions among those with uncertain migrant status. In addition, children in lower SEP areas could have access to suboptimal healthcare leading to poorer outcomes. We cannot rule out that the increased hazard of death observed in Black and Hispanic children after adjustment for area-level SEP could be partly explained by residual confounding by individual SEP. However, independently of SEP, the association with race/ethnicity could be related to racially/ethnically driven barriers to accessing adequate healthcare. Whilst it is difficult to unravel the mechanisms leading to this, implicit bias against minority groups in healthcare providers is well documented [34]. The presenting symptoms of CNS tumour can often be vague and non-specific, for example dizziness, vomiting, headache, altered gait, or changes in personality. Black or Hispanic children and those of lower SEP might be less likely to have such symptoms further investigated by healthcare workers. These barriers to seeking and receiving care for such symptoms may therefore delay diagnosis. Future analyses taking account of the pathway of care, not available in these data, might help better understand the mechanisms underlying these disparities.

\section{Conclusions}

This study shows strong survival disparities by race/ethnicity in 
children and adolescents diagnosed with CNS tumour in the US between 2000 and 2015. The findings parallel the large and growing body of evidence on outcome disparities by race/ethnicity in childhood and adolescent cancer in the US. Further work with reliable and complete information on tumour type, stage at diagnosis and treatment will be crucial to understanding the mechanisms by which these disparities occur. Further research into the healthcare access barriers faced by minority groups would also be crucial and together these could be the basis for healthcare policies aimed at eliminating these unacceptable survival inequalities.

\section{Contributor's statement}

Dr Mitchell conceptualized and designed the study, acquired and analysed the data, drafted the initial manuscript, and reviewed and revised the manuscript.

Drs Morris and Bonaventure conceptualized and designed the study, analysed the data and critically reviewed and revised the manuscript.

Drs Abrahão and Ellis critically reviewed and revised the manuscript.

All authors approved the final manuscript as submitted and agree to be accountable for all aspects of the work.

\section{Funding}

This research did not receive any specific grant from funding agencies in the public, commercial, or not-for-profit sectors.

\section{Declaration of Competing Interest}

The authors have no competing interests to declare.

\section{References}

[1] https://www.cancer.gov/about-cancer/understanding/statistics (Accessed 6th September 2019).

[2] S. Curtin, Brain Cancer death rates among children and teens aged 1-19 years, by sex and age group - united States, 2013-2015, MMWR Morb. Mortal. Wkly. Rep. 66 (2017) 461, https://doi.org/10.15585/mmwr.mm6617a5.

[3] E.W. Tai, K.C. Ward, A. Bonaventure, D.A. Siegel, M.P. Coleman, Survival among children diagnosed with acute lymphoblastic leukemia in the United States, by race and age, 2001 to 2009: Findings from the CONCORD-2 study, Cancer 123 (2017) 5178-5189, https://doi.org/10.1002/CNCR.30899.

[4] C. Allemani, T. Matsuda, V. Di Carlo, R. Harewood, M. Matz, M. Nikšić, A. Bonaventure, M. Valkov, et al., Global surveillance of trends in cancer survival 2000-14 (CONCORD-3): analysis of individual records for 37513025 patients diagnosed with one of 18 cancers from 322 population-based registries in 71 countries, Lancet (London, England) 391 (2018) 1023-1075, https://doi.org/10.1016/ S0140-6736(17)33326-3.

[5] B. Novakovic, U.S. Childhood cancer survival, 1973-1987, Med. Pediatr. Oncol. 23 (1994) 480-486 (Accessed November 30, 2017), http://www.ncbi.nlm.nih.gov/ pubmed/7935174.

[6] N.S. Kadan-Lottick, K.K. Ness, S. Bhatia, J.G. Gurney, Survival variability by race and ethnicity in childhood acute lymphoblastic leukemia, JAMA 290 (2003) 2008, https://doi.org/10.1001/jama.290.15.2008.

[7] R. Abrahão, D.Y. Lichtensztajn, R.C. Ribeiro, N.M. Marina, R.H. Keogh, R. MarcosGragera, S.L. Glaser, T.H.M. Keegan, Racial/ethnic and socioeconomic disparities in survival among children with acute lymphoblastic leukemia in California, 19882011: a population-based observational study, Pediatr. Blood Cancer 62 (2015) 1819-1825, https://doi.org/10.1002/pbc.25544.

[8] A.M. Linabery, J.A. Ross, Childhood and adolescent Cancer survival in the U.S. By race and ethnicity (Diagnostic period 1975-1999), Cancer 113 (2008) 2575, https://doi.org/10.1002/CNCR.23866.

[9] S. Gupta, M. Wilejto, J.D. Pole, A. Guttmann, L. Sung, Low socioeconomic status is associated with worse survival in children with Cancer: a systematic review, PLoS One 9 (2014) e89482, , https://doi.org/10.1371/journal.pone.0089482.

[10] H. Mogensen, K. Modig, G. Tettamanti, F. Erdmann, M. Heyman, M. Feychting, Survival after childhood cancer-social inequalities in high-income countries, Front. Oncol. 8 (2018), https://doi.org/10.3389/fonc.2018.00485.

[11] M.T. Austin, E. Hamilton, D. Zebda, H. Nguyen, J.M. Eberth, Y. Chang, L.S. Elting, D.I. Sandberg, Health disparities and impact on outcomes in children with primary central nervous system solid tumors, J. Neurosurg. Pediatr. 18 (2016) 585-593,
https://doi.org/10.3171/2016.5.PEDS15704.

[12] D.A. Siegel, J. Li, H. Ding, S.D. Singh, J.B. King, L.A. Pollack, Racial and ethnic differences in survival of pediatric patients with brain and central nervous system cancer in the United States, Pediatr. Blood Cancer 66 (2019) e27501, , https://doi. org/10.1002/pbc.27501.

[13] R.D. Kehm, L.G. Spector, J.N. Poynter, D.M. Vock, S.F. Altekruse, T.L. Osypuk, Does socioeconomic status account for racial and ethnic disparities in childhood cancer survival? Cancer 124 (2018) 4090-4097, https://doi.org/10.1002/cncr.31560.

[14] J.S. Barnholtz-Sloan, R.K. Severson, B. Stanton, M. Hamre, A.E. Sloan, Pediatric brain tumors in Non-Hispanics, Hispanics, African Americans and Asians: differences in survival after diagnosis, Cancer Causes Control 16 (2005) 587-592, https://doi.org/10.1007/s10552-004-7843-2.

[15] W.T. Curry, F.G. Barker, Racial, ethnic and socioeconomic disparities in the treatment of brain tumors, J. Neurooncol. 93 (2009) 25-39, https://doi.org/10.1007/ s11060-009-9840-5.

[16] F.M. Iwamoto, A.S. Reiner, K.S. Panageas, E.B. Elkin, L.E. Abrey, Patterns of care in elderly glioblastoma patients, Ann. Neurol. 64 (2008) 628-634, https://doi.org/10 1002/ana.21521.

[17] L.S. Schmidt, H. Nielsen, S. Schmiedel, C. Johansen, Social inequality and incidence of and survival from tumours of the central nervous system in a population-based study in Denmark, 1994-2003, Eur. J. Cancer 44 (2008) 2050-2057, https://doi. org/10.1016/j.ejca.2008.06.015.

[18] M. Adam, C.S. Rueegg, K. Schmidlin, A. Spoerri, F. Niggli, M. Grotzer, N.X. von der Weid, M. Egger, N. Probst-Hensch, M. Zwahlen, C.E. Kuehni, Socioeconomic disparities in childhood cancer survival in Switzerland, Int. J. Cancer 138 (2016) 2856-2866, https://doi.org/10.1002/ijc.30029.

[19] M. Son, J. Kim, J. Oh, I. Kawachi, Inequalities in childhood cancer mortality according to parental socioeconomic position: a birth cohort study in South Korea, Soc. Sci. Med. 72 (2011) 108-115, https://doi.org/10.1016/j.socscimed.2010.10 007.

[20] J.-H. Tseng, M.-Y. Tseng, Survival analysis of children with primary malignant brain tumors in England and Wales: a population-based study, Pediatr. Neurosurg. 42 (2006) 67-73, https://doi.org/10.1159/000090458.

[21] National Cancer Institute: Surveillance, Epidemiology, and End Results Program: Race recode changes for the 1973-2005 SEER research data (November 2007 submission) and later releases, (2019) (Accessed 6th September 2019), https://seer. cancer.gov/seerstat/variables/seer/race ethnicity/.

[22] NAACCR Race and Ethnicity Work Group, NAACCR Guideline for Enhancing Hispanic/Latino Identification: Revised NAACCR Hispanic/Latino Identification Algorithm [NHIA v2.2.1], Springfield(IL): North American Association of Central Cancer Registries, 2011 September.

[23] M. Yu, Z. Tatalovich, J.T. Gibson, K.A. Cronin, Using a composite index of socioeconomic status to investigate health disparities while protecting the confidentiality of cancer registry data, Cancer Causes Control 25 (2014) 81-92, https://doi.org/10. 1007/s10552-013-0310-1.

[24] K. Yost, C. Perkins, R. Cohen, C. Morris, W. Wright, Socioeconomic status and breast cancer incidence in California for different race/ethnic groups, Cancer Causes Control 12 (2001) 703-711, https://doi.org/10.1023/A:1011240019516.

[25] A. Fritz, C. Percy, A. Jack, K. Shanmugaratnam, L. Sobin, M. Parkin, S. Whelan, ICDO International Classification of Diseases for Oncology First Revision, (2013) (Accessed December 29, 2018), www.who.int.

[26] C. Zippin, D. Lum, B.F. Hankey, Completeness of hospital cancer case reporting from the SEER Program of the National Cancer Institute, Cancer 76 (1995) 2343-2350 (Accessed June 16, 2018), http://www.ncbi.nlm.nih.gov/pubmed/ 8635041

[27] N. Krieger, Overcoming the absence of socioeconomic data in medical records: validation and application of a census-based methodology, Am. J. Public Health 82 (1992) 703-710 (Accessed March 30, 2019), http://www.ncbi.nlm.nih.gov/ pubmed/1566949.

[28] L.X. Clegg, M.E. Reichman, B.F. Hankey, B.A. Miller, Y.D. Lin, N.J. Johnson, S.M. Schwartz, L. Bernstein, V.W. Chen, M.T. Goodman, S.L. Gomez, J.J. Graff, C.F. Lynch, C.C. Lin, B.K. Edwards, Quality of race, Hispanic ethnicity, and immigrant status in population-based cancer registry data: implications for health disparity studies, Cancer Causes Control 18 (2007) 177-187, https://doi.org/10 1007/s10552-006-0089-4.

[29] D.R. Williams, N. Priest, N.B. Anderson, Understanding associations among race, socioeconomic status, and health: patterns and prospects, Health Psychol. 35 (2016) 407-411, https://doi.org/10.1037/hea0000242.

[30] https://www.census.gov/quickfacts/fact/table/US/PST045217 (Accessed 6th September 2019).

[31] C.-H. Pui, J.M. Boyett, M.L. Hancock, C.B. Pratt, W.H. Meyer, W.M. Crist, Outcome of treatment for childhood cancer in black as compared with white children, JAMA 273 (1995) 633, https://doi.org/10.1001/jama.1995.03520320043039.

[32] M.J. Lund, M.T. Eliason, A.E. Haight, K.C. Ward, J.L. Young, R.D. Pentz, Racial/ ethnic diversity in children's oncology clinical trials, Cancer 115 (2009) 3808-3816, https://doi.org/10.1002/cncr.24437.

[33] J.M. Lee, X. Wang, R.P. Ojha, K.J. Johnson, The effect of health insurance on childhood cancer survival in the United States, Cancer 123 (2017) 4878-4885, https://doi.org/10.1002/cncr.30925.

[34] C. FitzGerald, S. Hurst, Implicit bias in healthcare professionals: a systematic review, BMC Med. Ethics 18 (2017) 19, https://doi.org/10.1186/s12910-017-0179-8. 